\title{
Design of a computer tool for the optimization of solid waste collection circuits
}

\author{
Nezha Haddoun $^{1, *}$, Driss Khomsi ${ }^{1}$ \\ ${ }^{1}$ Laboratory Analysis of Hydraulic Systems, Mohammadia School of Engineers, Mohammed V Rabat University, Morocco
}

\begin{abstract}
The optimization of solid waste collection circuits is one of the applications of the Capacited Arc Routing Problem. This article addresses this topic, summarizes and compares the different research and methods that have been used to solve this famous problem. And concluded with an effective method on which will be based the design of a computer optimization tool.
\end{abstract}

\section{Introduction}

The problem of solid waste collection is one of the most serious issues of concern to communities and garbage collection companies. It is a sector that represents a significant expense but receives little attention.

Indeed, our way of life and consumption leads us to produce more and more waste, which we have to manage. Logistics and waste transport are an essential element of this waste management in the same way as their methods of recovery or disposal, because of the mass and volumes concerned, the technical means put in place, the costs generated, or the energy consumption and emissions of pollutants generated.

The optimization of this management is the optimization of waste collection circuits. It will effectively reduce the cost of waste management and reduce the impact on the environment.

This complex problem is part of the Arc Routing Problems. The road network is represented by a graph in which each arc or edge (street) must be served at least once, the objective is to find a minimum cost cycle (or circuit) passing at least once through each arc or edge of the graph. This problem is well known in operations research under the name of the Chinese Postman Problem (CPP).

To be more realistic, several constraints are to be added to the Problem of the Arc Routing Problem. In the case of solid waste collection, it is the capacity constraints that must be taken into consideration. We are talking about the CARP " Capacited Arc Routing Problem ".

\section{Problem definition and formulation}

CARP was introduced by Golden and Wong (1981) [1]. The basic problem is usually defined in the literature on an undirected graph. The nodes include a depot where a fleet of identical vehicles of known capacity is based. Each edge has a crossing cost and a demand. Non-zero request edges must be crossed to be processed. CARP consists in determining a set of tours with a minimum total cost (this cost does not take into account the cost of ownership of vehicles), such as :

- Each tour, provided by a vehicle, begins and ends in the depot;

- Each edge can be crossed several times, by the same tour or different tours, but it must be processed by a single tour and in a single crossing;

- The sum of the requests processed (quantities of waste) by a tour must not exceed the capacity of the vehicle.

The basic problem can be formulated as follows:

$x_{i j k}=$ number of times that one crosses the edge $(\mathrm{vi}, \mathrm{vj})$ with the vehicle $\mathrm{k}$ without serving it.

$y_{i j k}=$

$\left\{\begin{array}{c}1 \text { if the edge }\left(v_{i}, v_{j}\right) \text { is served by the vehicule } k \\ 0 \text { if not }\end{array}\right.$

The wording of the problem is as follows

$$
\text { Minimize } \sum_{k=1}^{m} \sum_{(v i, v j) \in E} c_{i j}\left(x_{i j k}+y_{i j k}\right)
$$

Under the constraints:

$$
\begin{aligned}
& \sum_{k=1}^{m} y_{i j k}=1 \quad\left(\left(v_{i}, v_{j}\right) \in \mathrm{E}_{i} q_{i j}>0\right) \\
& \sum_{(v i, v j) \in E} q_{i j} y_{i j k} \leq \mathrm{Q} \quad\left(\mathrm{k}=1_{i \cdots \infty} \mathrm{m}\right) \\
& \sum_{(v i, v j) \in E(S)} x_{i j k}+\sum_{(v i, v j) \in E+(S)} y_{i j k} \geq 2 y_{h l k} \\
& \left(\mathrm{SCV}-\left\{v_{1}\right\} ; \mathrm{S} \neq \emptyset ; \mathrm{k}=1_{\text {consos }} \mathrm{m}\right) ; v_{h}, v_{l} \in S ; q_{\mathrm{hl}}>0 \text { ) } \\
& \text { (4) } \\
& \sum_{(v i, v j) \in E(S)} x_{i j k}+\sum_{(v i, v j) \in E+(S)} y_{i j k}=2 z_{k}^{S} \\
& \text { ( } \mathrm{SCV}-\left\{v_{1}\right\} ; \mathrm{S} \neq \emptyset ; \mathrm{k}=1 \text {,anso } \mathrm{m} \text { ) } \\
& z_{k}^{S} \geq 0 \text { integer } \quad\left(\mathrm{S} C \mathrm{~V}-\left\{v_{1}\right\} ; \mathrm{S} \neq \varnothing ; \mathrm{k}=1\right. \\
& x_{i j k} \geq 0 \text { integer } \quad\left(\left(v_{i}, v_{j}\right) \in \mathrm{E} ; \mathrm{k}=1, \ldots, \mathrm{m}\right) \\
& y_{i j k} \in\{0,1\} \quad\left(\left(v_{i}, v_{j}\right) \in \mathrm{E} ; \mathrm{k}=1, \ldots, \mathrm{m}\right)
\end{aligned}
$$

\footnotetext{
Corresponding author: haddoun.nezha@gmail.com
} 
With :

- The constraint (2) ensures that only edges with positive demand are served.

- Constraints on vehicle capacity are given by (3).

- The constraint (4) means that if $\left(\mathrm{v}_{\mathrm{h}}, \mathrm{v}_{\mathrm{l}}\right)$ is an edge served by the vehicle $\mathrm{k}$ such as $\mathrm{v}_{\mathrm{h}}, \mathrm{v}_{\mathrm{I}} € \mathrm{~S}$, then $\mathrm{S}$ must be connected to the other edges served by the vehicle $\mathrm{k}$.

- The constraint (5) ensures the return to the repository if you visit a subset of vertices $S$ that do not contain it by entering the integer variables $\mathrm{z}_{\mathrm{k}}^{\mathrm{s}}, \mathrm{k}=1, \ldots$, $\mathrm{m}, \mathrm{S} \mathrm{c} \mathrm{V}-\{\mathrm{v} 1\}$. This is because $\mathrm{S}$ must be connected an even number of times to $\mathrm{V}-\mathrm{S}$.

\section{Methods of resolution and literature}

CARP is an NP-hard problem, most resolution methods are based on heuristics and meta-heuristics. Nevertheless, exact algorithms have been proposed but can only solve small problems.

\subsection{Exact methods}

Exact methods have been based on CARP formulations in the form of linear integer programs (Golden and Wong (1981) [1]), using Branch and Bound algorithm (Hirabayashi et al. (1992) [2]), using the Cutting Planes (Belenguer and Benavent Method (2003) [3]), transforming the problem into a Node Routing Problem (Baldacci and Maniezzo (2006) [4] and Longo et al. (2006) [5]) etc. but these methods can only solve problems of limited size.

However, heuristics and metaheuristics remain the most effective methods for dealing with the most complex networks, these methods make it possible to give correct solutions to the problem even if they do not guarantee optimality.

\subsection{Heuristics}

The most used heuristics to solve the CARP are:

- The Algorithm Construct and Strike which was developed by Christofides (1973)

- Augment Merge Algorithm proposed by Golden and Wong (1981)

- Path Scanning algorithm presented by Golden et al (1983)

- Parallel insertion algorithm developed by Chapleau et al (1984)

- Route First, Cluster Second Algorithm developed by Ulusoy (1985)

- First-route second cluster algorithm Proposed by Benavent et al (1990)

\subsection{Metaheuristics}

Metaheuristics are powerful and evolved heuristics. The metaheuristics published for the CARP are:

- Tabou Search method (TS) : introduced by Fred W. Glover in 1986, it is a local search method combined with a set of techniques to avoid being trapped in a local minimum or the repetition of 'a cycle. This method has been applied to CARP by Hertz et al. (2000) [6] and Brandao and Eglese (2008) [7].

- Simulated Annealing method (SA): a combinatorial optimization method derived from physics inspired by steelmaking techniques to cool the metal: We heat a metal and then let it cool slowly, which allows to increase the degree of freedom of the atoms in order to reach a new dynamic state. Based on this principle, the simulated annealing method increases the degree of freedom by allowing degradation of the solution. The idea is to start from an initial solution chosen at random and to test if it respects all the constraints. If this is not the case, we slightly modify the solution so that it respects them. Then we examine the neighboring solutions. In order to be able to exit possibly from the local optimum, one accepts a degradation of the solution in a probability inversely proportional to the amplitude of this degradation. Thus, the greater the degradation is less the chance to retain this solution is high. Then, as one moves forward in time, it becomes more and more unlikely to accept degradation. The fact that a point can be accepted sometimes even if it gives a value of the function greater than the previous value allows the algorithm to leave local minima and possibly converge towards the global minimum. The algorithm ends when a predefined stopping criterion is reached.

- Genetic Algorithms (GA): Genetic algorithms are exploration algorithms based on the mechanisms of natural selection and genetics. They use both the principles of survival of the most suitable structures, and random information exchanges, sometimes guided, to form an exploration algorithm that has some of the characteristics of human exploration. This method was developed by John Holland in 1975. It was later formalized by Goldberg (1989) to be applied to solving optimization problems.

An GA models the process of collective evolution of a population of individuals to adapt to an environment. Each individual will represent not only one point in the solution space of the problem, but also contain the current knowledge of the individual in relation to the environment. The main concern of GA research is to improve the robustness and the balance between performance and cost necessary for survival in many different environments by applying three operators: selection, crossing and mutation.

The procedure of the genetic algorithm is described as follows:

- Generate a population of solutions

- As long as the stopping criterion is not met:

o Select in the current population all parents

o randomly classify all parents in pairs and apply a cross to produce the child vectors

o Apply a mutation, that is, a random change, to a random number of offspring

o Assess the offspring

o Create the new population from the previous population and children 
o Eventually, remove individuals with poorer performance.

The procedure usually ends when the number of fixed iterations, here the number of generation created, is reached or when the population can not be improved. The first genetic algorithm to solve the CARP was proposed by Lacomme et al (2001)[8]. In 2002 this algorithm was extended to treat ECARP (Extended CARP), SCARP (Stochastic CARP) and PCARP (Periodic CARP) by Wahiba Ramdane Cherif [9].

In 2018, Arakaki et al. proposed a hybrid genetic algorithm to solve the problem of open CARP [10].

- Ant Colony Optimization (ACO): This is a recent algorithm that has been proposed for the first time by Dorigo et al. (1996) [11] to solve the Traveling Salesman Problem. This method is inspired by the behavior of ants. These almost blind insects still manage to find the shortest way between their starting point and food through a form of learning and a means of communication: the pheromone. Pheromone is a substance deposited in varying quantities by a moving ant. Thus, the more ants use the same path, the more pheromones there will be, and the more likely it is that the next ants will pass.

To solve the CARP, the first ant algorithm was formulated by Lacomme et al (2003) [12], their work was based on the use of constructive heuristics to provide three good initial solutions and the improvement of local search solutions at the end of each iteration. This method has proved to be competitive with the best published taboo method [6] but not as effective as the genetic algorithm of Lacomme et al.[8].

In 2009, Kansou et al. addressed the CARP problem with several depots (MD-CARP) [13]. They developed two ant colony approaches: a hybrid approach with two steps : optimizing the order of tasks for insertion by optimizing ant colonies and insertion of tasks by a heuristic backup method. A generalization of the Ulusoy method is integrated into the optimization of the ant colony in the second approach. The results show the performance of the proposed methods.

Santos et al (2010) presented a metaheuristic for CARP based on ant colony optimization [14]. Changes was introduced for various components of ant colony methaheuristics mainly to the "initial population", the "ant decision rule" and the "local search procedure".

In 2011, Chen et al. developed a hybrid ant colony optimization algorithm to solve ECARP [15]. The algorithm was tested on four sets of reference problems containing a total of 87 instances with up to 140 nodes and 380 arcs. The results showed the effectiveness of this method.

In 2012, Boukachour et al. applied ant colony optimization to solve the waste collection problem [16]. An algorithm was developed and the best solution obtained is then improved by two local search heuristics.

\section{Latest results and comparison}

The last publication dealing with the problem of solid waste collection is the one that reflects at most the real case. This method, proposed by Tirkolaee et al. (2018) [17], brings together several constraints: capacity, multiple displacements, uncertainty on the demand as well as the working time of the drivers and the crew. The objective is to determine the optimal number of vehicles and the optimal route for each vehicle.

To solve this problem, a heuristic algorithm has been proposed to generate initial solutions (200 solutions) then a simulated annealing algorithm is applied to provide the best possible solutions.

This method yielded good results compared to the exact results provided by the CPLEX solver in a much shorter execution time.

Nevertheless, this method remains limited because it is only valid for non-directed graphs.

So, this research can still be pushed to deal with the case of mixed graphs. Also, solving the problem based on a genetic algorithm or an ant colony algorithm may be more efficient than the simulated annealing algorithm because the latter is based on the choice of several parameters taken empirically, such as the initial temperature, the temperature decay law, the stopping criteria.

\section{Method chosen for the development of the computer tool}

There are several optimization software for solid waste collection circuits (Operations Planner, Exeo Solutions, Solver Tower, Bluekaisen Residential Waste ...). The first optimization software was content to draw ideal tours.

Today, they offer many other features: dashboards, guidance and GPS tracking, recording anomalies, terminals acting as dictaphone or cameras, drawing schedules, etc.

Our tool will be more updated (based on the latest optimization methods) and will therefore give better solutions than the software that is marketed which means more gains.

This tool will be based on a study that is a succession of the last study carried out (same constraints) but using the genetic algorithms. This method has demonstrated, according to the literature, its ability to produce better results than those obtained by simulated annealing.

\section{Conclusion}

This article provides an updated bibliographic synthesis of Capacited Arc Routing Problem with the aim of solving the problem of optimizing solid waste collection circuits. It discusses the various studies carried out in the field and displays the latest results obtained.

This article also discusses the latest research, which is the most realistic because it brings together a large 
number of real constraints, cites the limitations of this study and shows that it can be further pushed to give better results (case of a mixed graph and application of genetic algorithms).

\section{References}

[1] BL Golden, RT Wong, (1981). 'Capacitated arc routing problems",

[2] R. Hirabayashi, N. Nishida, Y. Saruwatari, Tour Construction Algorithm For The Capacitated Arc Routing Problem, Asia Pacific Journal of Operational Research, Vol 9 (Issue 2), (January 1992)

[3] J.M. Belenguer, E. Benavent, A Cutting Plane Algorithm For The Capacitated Arc Routing Problem, Computers \& Operations Research, Vol.30 (Issue 5):705-728, (April 2003)

[4] R. Baldacci, V. Maniezzo, Exact Methods Based On Node Routing Formulations For Undirected Arc Routing Problems, Networks, Vol. 47(Issue1):5260, (January 2006)

[5] H. Longo, M.P. de Aragão, E. Uchoa, Solving Capacitated Arc Routing Problems Using A Transformation To The CVRP, Computers \& Operations Research, Vol. 33 (Issue 6): 1823-1837, (June 2006)

[6] A. Hertz, G. Laporte, M. Mittaz, A Tabu Search Heuristic For The Capacitated Arc Routing Problem, Operations Research, Vol.48 (Issue 1), (February 2000)

[7] J. Brandão, R. Eglese, A Deterministic Tabu Search Algorithm For The Capacitated Arc Routing Problem, Computers \& Operations Research, Vol. 35 (Issue 4): 1112-1126, (April 2008)

[8] P. Lacomme, C. Prins, W.R. Cherif, A Genetic Algorithm for the Capacitated Arc Routing Problem and Its Extensions (Applications of evolutionary computing, Evo Workshops, 2001, pp. 473-483) (2001)

[9] W.R. Cherif, Problèmes d'optimisation en tournées sur arcs, Ph.D. dissertation, Production management, Doctoral School Sciences for the Engineer, Troyes, Aube, (2002)

[10] R.K. Arakaki, F. L.Usberti, Hybrid Genetic Algorithm For The Open Capacitated Arc Routing Problem, Computers \& Operations Research, Vol. 90: 221-231, (February 2018)

[11] M. Dorigo, V. Maniezzo, A. Colorni, The ant system: Optimization by a colony of cooperating agents, IEEE Transactions on Systems Man and Cybernetics, Vol. 26 (Issue 1):29-41, (January 2002)

[12] P. Lacomme, C. Prins, A. Tanguy, Optimisation par colonies de fourmis pour les tournées sur arcs, 4th Francophone Conference of Modelling and Simulation "Organization and Conduct of Activities in Industry and Services" MOSIM'03, Toulouse, France, from 23 to 25 (April 2003)
[13] A. Kansou, A. Yassine, Ant Colony System for the Periodic Capacitated Arc Routing Problem, International Network Optimization, (2009)

[14] L. Santos, J.C. Rodrigues, J.R. Current, An Improved Ant Colony Optimization Based Algorithm For The Capacitated Arc Routing Problem, Transportation Research Part B Methodological, Vol. 44 (Issue 2): 246-266, (February 2010)

[15] L. Xing, P. Rohlfshagen, Y.W. Chen, X. Yao, A Hybrid Ant Colony Optimization Algorithm for the Extended Capacitated Arc Routing Problem, IEEE transactions on systems, man, and cybernetics. Part B, Cybernetics: a publication of the IEEE Systems, Man, and Cybernetics Society, Vol. 41 (Issue 4), (February 2011)

[16] D. Btissam, T Lamoudan, J.Boukachour, E.A. Ahmed, Un algorithme hybride pour la résolution du problème de collecte des déchets, 1st International IEEE Conference on Logistics Operations Management, Higher Institute of Logistics Studies, Havre, France, (October 2012)

[17] E.B. Tirkolaee , I. Mahdavi, M.M.S Esfahani, A Robust Periodic Capacitated Arc Routing Problem For Urban Waste Collection Considering Drivers And Crew's Working Time, Waste Management, Vol. 76: 138-146, (June 2018) 